\title{
Diabetes mellitus tipo 2: qual o papel da insulina na expressão de NF-kappaB, PPAR $\gamma$ e CD36?
}

\author{
Type 2 diabetes: What is the role of insulin in \\ NF-kappaB, PPAR $\gamma$ and CD36 expression?
}

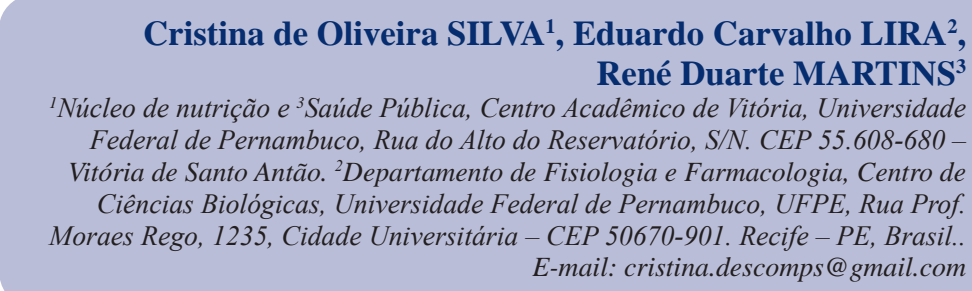

\section{ABSTRACT}

In both type II diabetes and insulin-resistance syndromes, vascular complications result from a set of atherogenic processes, involving chronic hyperglycemia, excessive protein glycation (AGEs), nuclear factor kappa-B (NF-kappaB) activation associated with inflammatory cytokines superexpression, oxidative stress altering LDL and scavenger receptor CD36 expression. The contribution of hyperinsulinaemia in this sequence is not completely elucidated. This review adresses the modulation of NF-kappaB, PPAR gamma (PPAR $\gamma$ ) and CD36 protein expression by insulin in rat aortic vascular smooth muscle cells (VSMCs) stimulated by AGEs

Key Words: Type 2 Diabetes; advanced glycation end products; oxidative stress.

\section{RESUMO}

No diabetes mellitus tipo 2 (DM2) e na síndrome de resistência à insulina, as complicações cardiovasculares resultam de um conjunto de processos aterogênicos envolvendo hiperglicemia crônica, excessiva glicação de proteínas (AGEs), ativação do fator nuclear kappa $\mathrm{B}(\mathrm{NK \kappa B})$ associada com o aumento da expressão de citocinas inflamatórias e estresse oxidativo, observando-se ainda alteração de LDL e expressão do receptor de scavenger CD36. A contribuição da hiperinsulinemia nesta sequência não é completamente elucidada. Nesta revisão, relata-se como a insulina pode modular a expressão proteica de NFKB, PPAR gama (PPAR $\gamma$ ) e CD36 em células da musculatura lisa vascular (CMLV) da aorta de ratos estimuladas pelos AGE.

Palavras Chave: Diabetes Mellitus tipo 2; produtos finais de glicação avançada; estresse oxidativo

\section{INTRODUÇÃO}

A exposição prolongada à hiperglicemia é reconhecida como o principal fator responsável pela fisiopatologia das complicações diabéticas (1). Entre as hipóteses que tentam explicar como a hiperglicemia crônica determina os danos celulares e teciduais observados no diabetes mellitus (DM), a formação dos produtos finais da glicação avançada, também chamados AGE, é considerada uma das mais importantes. Os AGE constituem uma classe de moléculas heterogêneas formadas a partir de reações ami- nocarbonila de natureza não-enzimática, que no estado hiperglicêmico ocorre de forma acelerada e são capazes de modificar, irreversivelmente, as propriedades químicas e funcionais de várias estruturas biológicas, dentre elas, os fosfolipídios de membrana, proteínas e ácidos nucleicos (2). Embora parte dos mecanismos que levam às complicações diabéticas permaneça pouco compreendido, a formação dos AGE, o desenvolvimento do estresse oxidativo e a ativação da proteína quinase $\mathrm{C}$ (PKC) são mecanismos relacionados à hiperglicemia crônica e as alterações morfológicas e funcionais do endotélio no diabetes $(3,4)$. 
Os receptores para AGE (RAGE) fazem parte da superfamília de receptores para imunoglobulinas inicialmente identificados em células endoteliais de pulmão bovino (5). É uma proteína de $35 \mathrm{KDa}$ expressa em diferentes tecidos, tais como células ósseas, células mononucleares, linfócitos, células da musculatura lisa vascular (CMLV), coração e neurônios (5-8). São bem demonstradas as alterações vasculares importantes induzidas pela ativação dos RAGE como parte dos mecanismos envolvidos nas complicações diabética; entretanto, se isto ocorre a partir da ligação dos AGE aos RAGE ainda é alvo de intenso debate. Isto porque o RAGE pode ser ativado por diferentes ligantes pró-inflamatórios, como a proteínas de alta mobilidade do grupo 1 (HMGB1) ou anfoterina (9), S100/calgranulina (10), proteína $\boldsymbol{\beta}$-amiloide (5), fosfatidilserina (11) e produtos avançados de oxidação de proteínas (AOPP) (12). De modo muito interessante, Schmidt et al. (1999) demonstraram que a ligação de AGE-RAGE determina a ativação de vias inflamatórias e a produção de fatores de adesão celular envolvidos no desenvolvimento da neuropatia diabética. Há outras isoformas destes receptores, como o RAGE solúvel (sRAGE), cuja ativação não determina alterações celulares, mas contribuem para a depuração plasmáticas dos AGE (13).

$\mathrm{O}$ que torna ainda mais complexo os mecanismos de ação dos AGEs é que também são ligantes para outros receptores, como o CD36, receptor scavenger da classe B, que está envolvido na metabolização dessas moléculas no plasma, assim como exerce importante função na indução do estresse oxidativo na célula. Além destes, os AGE também são reconhecidos pelos receptores scavenger classe $\mathrm{E}$, lectin-like oxidized LDL receptor-1 (LOX1), fasciclina, epidermal growth factor-like (EGF), laminin-type epidermal growth factor-like (LE), link domain-containing scavenger receptor-1 e 2 (FEEL-1 e FEEL-2) $(14,15)$.

A caracterização das interações AGEs-RAGE nas lesões vasculares, foram observadas em ratos diabéticos sensibilizados à aterogênese pela supressão da apolipoproteína $\mathrm{E}$ (apoE-null), mostrando que o tratamento após seis semanas com uma forma C-truncada do receptor RAGE (fator inibitório) diminuiu a frequência de lesões da aorta (13). Este efeito foi atribuído ao provável sequestro dos ligantes de RAGE que impedem a transdução de sinal (16).

A interação AGE-RAGE favorece a síntese de espécies reativas de oxigênio (ERO) e o aumento da permeabilidade da barreira endotelial (17), o que pode induzir o estresse oxidativo e ativar o fator de transcrição nuclear kappa B (NFkB) (18). A via de transdução do sinal dependente de RAGE associa a ativação de quinases pertencentes à família das proteínas quinase ativada por mitógeno (MAP-quinases) e a translocação de NFкB para o núcleo, o que estimula a transcrição de vários genes-alvo associados ao desenvolvimento da inflamação $(19,20)$. Evidências recentes também demonstraram a importância dos receptores ativados por proliferadores de peroxissoma (PPAR) no controle de diversos processos biológicos que regulam a homeostase da glicose, o metabolismo de lipídeos e a inflamação, cuja importância é crucial nas doenças cardiovasculares. Estudos sugerem que o PPAR- $\gamma$ tem sua expressão modulada por AGE em lesões ateroscleróticas (21).

As complicações vasculares são as maiores causas de morbimortalidade em pacientes diabéticos. O desenvolvimento das doenças micro e macrovasculares invariavelmente se iniciam pela disfunção endotelial; entretanto, seus mecanismos não são totalmente compreendidos. Os prejuízos ocasionados ao endotélio vascular parecem ser um evento precoce na patogênese das complicações vasculares do diabetes mellitus tipo 2 (DM2) e reflete a presença de um fenótipo propenso à aterogênese, caracterizando-se pela perda das propriedades do endotélio, isto é, alteração na síntese de proteínas, aumento do tônus vascular, aumento da permeabilidade vascular e aquisição de atividade pró-trombótica e antifibrinolítica (22). Os PPAR podem afetar a formação das células espumosas, modulando a resposta inflamatória e influenciando a estabilidade da placa aterosclerótica (24). Neste sentido, um elo que é explorado, envolvendo a ação dos PPAR nessa doença, é o efeito anti-inflamatório exercido por alguns ativadores do PPAR- $\gamma$ e do PPAR- $\alpha$, como as glitazonas e os fibratos, com potenciais implicações no tratamento da aterosclerose (25).

\section{O fator de transcrição nuclear $\mathrm{kB}$}

Descoberto em 1986, NFкB é um heterodímero constituído de duas subunidades proteicas: RelA (p65), RelB, c-Rel, NFkB1 (p50) e NFкB2 (p52). Essas proteínas estruturalmente homólogas formam diferentes homo e heterodímeros por meio de seus domínios amino terminal (Rel Homology Domains, RHD) denominados $\mathrm{NF \kappa B}$. O fator de transcrição NFкB uma vez ativado, liga-se a uma sequência de 10 pares de bases na região promotora do gene que codifica a cadeia leve kappa das moléculas de anticorpo das células B provendo a transcrição de genes envolvidos em cascatas inflamatórias e geração do estresse oxidativo, condições determinantes na fisiopatologia do DM (26).

A ativação do NFкB é controlada pela família de inibidores (denominados IkB), que estão ligados ao dímero do $\mathrm{NF \kappa B}$, e inibem sua função, mantendo o complexo inativo no citoplasma celular. Diversos estímulos ativam o NFKB, como a fosforilação e a ativação 
do complexo IkB quinase (IKK). Esse complexo consiste de duas subunidades (dois heterodímeros: IKKa e IKK $\beta$ ) que fazem parte da subunidade IKK $\gamma$.

Na via canônica de ativação do NFKB, o complexo IKK ativado fosforila especificamente os I $\mathrm{KB} \alpha$, provavelmente pela I $\kappa B \beta$, tornando-o alvo de degradação pelo proteassoma (27). A partir disto, o NFкB é translocado ao núcleo, onde modula a expressão de diferentes genes. A ativação do NFkB pode ser iniciada por uma variedade de alterações fisiopatológicas, como aumento na quantidade de citocinas circulantes e na expressão de moléculas de adesão $(28,29)$. É importante ressaltar que a ativação de RAGE produz o estresse oxidativo e ativa $\mathrm{NF \kappa B}$ in vitro e in vivo $(30,18)$. A via de transdução do sinal dependente de RAGE envolve a via das proteínas MAP-kinases (Proteína Quinase Ativada por Mitógeno). Em contraste com outros receptores, a ativação celular mediada por RAGE inclui aumento da expressão do próprio RAGE. Esse mecanismos de retroalimentação positiva caracterizados pela interação do receptor com o ligante seguido pelo aumento da expressão do próprio receptor, sugerem ao RAGE o papel de fator de propagação e perpetuação do processo inflamatório (10). No caso do DM, a ativação do complexo NFKB, contribui com maior expressão gênica das moléculas envolvidas no processo inflamatório (31). Ademais, estudo mais recente indicou que uma ativação de NFkB por TNF- $\alpha$, induz a uma ativação de IKK $\beta$ que amplificaria o estresse oxidativo, levando a uma disfunção endotelial no DM-2 (29), o que sugere a participação de mais de uma via de sinalização intracelular envolvida no desenvolvido do estado inflamatório característico do DM.

Vários estímulos promovem a ativação de $\mathrm{NF \kappa B}$ portanto, não é surpreendente que sejam propostos mecanismos diferenciados para interpretar os eventos que precedem a fosforilação $\mathrm{IkB} \alpha$. O estresse oxidativo é um fator comum a todos estes estímulos, gerando espécies reativas de oxigênio (ERO) que induz a transcrição de um programa gênico relacionado à inflamação $(31,32)$. Foi demonstrado que em pacientes com DM1 com glicemia não controlada, ocorre maior ativação do NFKB em monócitos e uma correlação positiva com o estresse oxidativo (33). Contudo, em pacientes com DM2, além da maior ativação do NFאB ocorre diminuição dos níveis de glutationa redutase (34), o que reitera o aumento do estresse oxidativo nesta situação.

Devido aos diferentes modelos e estímulos utilizados para as interações entre estresse oxidativo e via do NFKB, há controvérsias sobre os mecanismos propostos para interpretar os eventos que precedem a última etapa da fosforilação de IкB. Tem sido mostrado que o peróxido de hidrogênio $\left(\mathrm{H}_{2} \mathrm{O}_{2}\right)$ ativa diretamente NFkB. De modo oposto, moléculas antioxidantes como a $\mathrm{N}$-acetilcisteína (NAC), vitaminas C e E, as selenoproteínas e o ácido lipoico também ativam NFkB. Entretanto, a ativação de NFkB pelo TNF- $\alpha$ ou pela LDL-ox é bloqueada pela NAC (35), o que sugere que o uso de antioxidantes atenuam o estado inflamatório no DM, o que permite especular uma via de estimulação independente de IKK. Ademais, em células T (junkat) e células endoteliais (U937), quando o estresse oxidativo é provocado pela hipóxia seguida de reoxigenarão ou pelo uso do pervanadato, a ativação de NFkB ocorreria por meio da fosforilação da tirosina I $k B$ e não pela via canônica, que é a fosforilação de serinas (36).

A insulina também parece ter papel modulador na expressão proteica de $\mathrm{NF \kappa B}$, embora ainda sejam escassos os trabalhos relatados na literatura. Em pacientes obesos, a insulina inibe a expressão NFкB em células mononucleadas (37). Incubações de CMLV da aorta de ratos Goto-Kakizaki (GK), um modelo genético de diabetes sem obesidade, com albumina bovina glicosilada (AGE-BSA) durante 24 horas, aumentou a expressão protéica do $\mathrm{NF \kappa B}$, o que foi atenuado na presença de insulina (38). Estes achados mostram que a insulina pode diminuir a incidência de alterações nas CMLV de ratos GK por meio da redução da expressão proteica do NFкB. Paradoxalmente, em estudos utilizando CMLV da aorta bovina incubada com insulina, na presença de AGE-BSA, ocorreu aumento da ativação do NFkB (39). Isto se deve possivelmente ao sistema utilizado para quantificação da proteína, que neste caso foi realizada por sistemas de genes reporter, que fornecem apenas uma estimativa indireta da atividade transcricional do vetor "reporter" que sintetiza a proteína, mas não reflete o nível celular do NFKB. Esta discrepância também pode ser explicada pelas diferenças entre as propriedades intrínsecas das CMLV de diferentes espécies e/ou diferenças no isolamento e preparação, bem como condições de cultura e influência do estado diabético.

\section{Os receptores ativados por proliferadores de peroxissoma}

Os PPAR são fatores de transcrição da família de receptores nucleares, caracterizados principalmente pela sua distribuição nos tecidos e por sua função metabólica. Formam heterodímeros com receptor do ácido cis-9-retinóico (RXR) e regulam a expressão gênica por se ligarem ao o elemento responsivo ao PPAR (PPREPeroxisome Proliferator Response Element) na região promotora dos genes-alvo $(40,41)$, os quais são ativados em resposta a ácidos graxos e derivados, incluindo uma variedade de eicosanóides e prostaglandinas, que têm papel chave no processo inflamatório e na resposta imunológica. Os ácidos graxos per se, têm capacidade de se ligar a todas as isoformas de PPAR (42). O complexo 
PPAR-RXR também pode se acoplar ao elemento responsivo em genes-alvo na ausência de ligantes e recrutar complexos corepressores que mediam a repressão ativa de genes (43).

As principais isoformas de PPAR identificadas até o momento são PPAR $\alpha, \operatorname{PPAR} \delta / \beta$ e PPAR $\gamma$. A ativação de PPAR $\gamma$ pode diminuir a progressão da aterosclerose e aumentar a sensibilidade à insulina, o que o torna um alvo terapêutico promissor no tratamento do DM2 e das dislipidemias. Vários estudos relatam que ligantes do PPAR $\gamma$ podem reduzir a liberação de citocinas inflamatórias (TNF- $\alpha$, IL-6 e IL-1 $\beta$ ) em monócitos, assim como a isoforma indutível da sintase do óxido nítrico e a expressão de metaloproteinases-9, as quais estão implicadas na desestabilização das placas ateroscleróticas $(44,45)$. Esses efeitos resultariam da inibição das vias dos fatores de transcrição da proteína ativadora -1 (AP-1) e NFkB, fatores de transcrição que aumentam a expressão de TNF- $\alpha$ e IL-6. Foi sugerido que a prostaglandina J2 (15d-PGJ2), um ligante natural do PPAR $\gamma$, exerce seu efeito anti-inflamatório pela inibição do NFkB $(46,47)$. Incubações de CMLV, oriundas de ratos GK e Wistar, não diabéticos, com AGE-BSA na presença de insulina aumenta a expressão proteica de PPAR $\gamma$ (38). Este efeito é consistente com o uso terapêutico da insulina e agonistas do PPAR $\gamma$ no DM (48).

Embora em modelos animais o PPAR $\delta / \beta$ apresentem efeitos anti-inflamatórios e anti-aterogênicos, o seu uso clínico ainda não está estabelecido (49). É importante ressaltar, que a ativação do PPAR $\gamma$ em macrófagos leva à expressão do $\mathrm{CD} 36$, responsável pela captação de LDL-ox, sendo este evento fundamental na diferenciação em macrófagos com características de célula espumosa (50). As LDL-ox contêm altas concentrações de 9- e 13- HODE (ácido hidroxiocatadecadienoico), ativadores do PPAR $\gamma$, desencadeando a expressão de receptores scavenger, como CD36 que promovem a internalização de moléculas de LDL-ox. A estimulação de LDL-ox induz a ativação do NFKB em macrófagos (51), por meio de um mecanismo que é dependente de CD36 e PKC (52). Além disso, o NFKB também estimula a secreção de citocinas inflamatórias como TNF $\alpha / \beta$, IL-1 $\beta$, IL-6 e interferon $\beta$ e $\gamma$, iniciando um sistema de retroalimentação positiva que promoveria a formação de células espumosas através da ativação de PPAR $\gamma(53)$.

Estudos realizados por Chinetti et al. (2001), mostraram que PPAR $\gamma$ e PPAR $\alpha$ não induzem formação de células espumosas a partir de monócitos circulantes agregados à parede arterial e diferenciados em macrófagos (54), apesar dos seus efeitos sobre a expressão de CD36. Nesse contexto, foi verificado que as tiazolidinadionas (TZD) estimulam a expressão de CD36, mas reduzem a expressão de SR-A (Scavenger Receptor A). Essas ações são compensatórias e exercem um efeito nulo sobre os valores intracelulares de ésteres de colesterol. Outro mecanismo pelo qual PPAR $\gamma$ intervém na homeostase do colesterol em macrófagos é por meio da indução de ABCA1 (ATP-binding cassette transporter A1), um transportador de colesterol livre, permitindo a saída de colesterol dos macrófagos em uma das primeiras etapas de transporte reverso de colesterol. Desta forma, os ésteres de colesterol armazenados, que se encontram em equilíbrio dinâmico com o colesterol livre, submetidos a um processo contínuo de hidrólise e re-esterificação, podem ser transferidos por um transportador de colesterol a um aceptor de colesterol (apo A-I). Este mecanismo envolve o receptor LXRa, corroborando a ideia de transporte reverso do colesterol de volta para o fígado $(55,56)$. Portanto, os agonistas de PPAR $\gamma$, apesar de incrementarem a expressão de um fator pró-aterogênico, produzem outros efeitos que causam uma ação anti-aterogênica.

\section{O receptor scavenger CD36}

O papel do CD36 em fenômenos associados à aterogênese e à resistência à insulina é foco de inúmeros estudos, porém seus mecanismos não são bem conhecidos. O CD36 é mencionado como o gene da resistência à insulina (57), mas o assunto é complexo, visto que animais knockout (CD36-/-) não são protegidos contra o diabetes. Aumento na expressão de CD36 em ratos transgênicos (MCK-CD36), por sua vez, não os torna diabéticos (58). Esta ambiguidade revela a multiplicidade funcional de CD36. Também é conhecido que a resistência à insulina é acompanhada de aumento do CD36 em macrófagos oriundos de ratos obesos diabéticos (ob/ob), e parece resultar da redução da degradação de proteínas. Esta anomalia é decorrente de prejuízos na via de transdução da insulina, uma vez que o uso de um inibidor da fosfoinositol-3-quinase (PI3K) aumenta a expressão do CD36 (59). As possibilidades de se interferir na expressão desse receptor, notadamente por ações mediadas por agonistas do PPAR $\gamma$, parecem ser importantes na patogênese de doenças cardiovasculares, em particular nos processos envolvendo mecanismos inflamatórios (60).

Em CMLV da aorta de ratos GK foi observado que a expressão proteica de CD36 é maior quando comparada a ratos não-diabéticos. Entretanto, quando essas células foram incubadas na presença de AGE-BSA, essa expressão foi aumentada.Na presença de insulina, a expressão de CD36 é reduzida ou suprimida nas células oriundas de ratos não-diabéticos, permanecendo inalterada nas oriundas de ratos GK, corroborando a hipótese de que esses últimos têm um defeito na via de sinalização da insulina (61). Estudos adicionais são necessários para melhor caracterizar a ação moduladora da insulina sobre a expressão de CD36 em modelos animais. 


\section{CONCLUSÃO}

Considerando a ampla rede de sinalização intracelular envolvida na rota inflamatória implicada no Diabetes Mellitus, na aterosclerose e em doenças crônicas, a modulação da resposta à insulina na expressão de NF $\kappa$ B, PPAR $\gamma$ e CD36 pode ser considerada como favorável à proteção vascular. Na presença de AGE, a insulina aumenta a expressão proteica de PPAR $\gamma, o$ que diminui a resposta inflamatória vascular e inibe a atividade de fatores transcricionais, como o NFкB. Deste modo, acredita-se que a insulina atenua o estado inflamatório no DM induzido pelos $\mathrm{AGE}$ ao inibir a atividade transcricional do NFкB. Em conjunto, os dados indicam os PPAR como alvos terapêuticos importantes para o desenvolvimento de novos fármacos que possam atenuar ou prevenir a disfunção endotelial e suas consequências.

\section{REFERÊNCIAS}

1. Laakso M. Hyperglycemia and cardiovascular disease in type 2 diabetes. Diabetes, 1999. 48(5):937-42.

2. Brownlee M. The pathological implications of protein glycation. Clin Invest Med, 1995. 18(4):275-81.

3. Baynes J. Role of oxidative stress in development of complications in diabetes. Diabetes, 1991. 40(4):405-12.

4. Nishikawa T, et al. Normalizing mitochondrial superoxide production blocks three pathways of hyerglycaemic damage. Nature, 2000. 404 (6779):787-90.

5. Neeper M, Schmidt AM, Brett J, Yan SD, Wang F, Pan YC, Elliston K, Stern D, Shaw A. Cloning and expression of a cell surface receptor for advanced glycosylation end products of proteins. J Biol Chem, 1992. 267(21):14998-5004.

6. Barlovic DP, Soro-Paavonen A, Jandeleit-Dahm KA. RAGE biology, atherosclerosis and diabetes. Clin Sci (Lond), 2011. 121(2):43-55

7. Nedié O, Rattan SI, Grune T, Trougakos IP. Molecular effects of advanced glycation end products on cell signalling pathways, ageing and pathophysiology. Free Radic Res, 2013. 47(suppl 1):28-38.

8. Xie J, Méndez JD, Méndez-Valenzuela V, Aguilar-Hernández MM. Cellular signalling of the receptor for advanced glycation end products (RAGE). Cell Signal, 2013. 25(11):2185-97.

9. Hori O, et al. The receptor for advanced glycation end products (RAGE) is a cellular binding site for amphoterin. Mediation of neurite outgrowth and co-expression of rage and amphoterin in the developing nervous system. J Biol Chem, 1995. 270(43):25752-61.

10. Hofmann MA, et al. RAGE mediates a novel proinflammatory axis: a central cell surface receptor for S100/calgranulin polypeptides. Cell, 1999. 97(7):889-901.

11. He M, et al. Receptor for advanced glycation end products binds to phosphatidylserine and assists in the clearance of apoptotic cells. EMBO Rep, 2011. 12(4):358-64.

12. Zhou LL, et al. The receptor of advanced glycation end products plays a central role in advanced oxidation protein products-induced podocyte apoptosis. Kidney Int, 2012. 82(7):759-70.
13. Park L, Raman KG, Lee KJ, Yan L, Ferran LJ, Chows WS. Suppression of accelerated diabetic atherosclerosis by soluble receptor for AGE (sRAGE). Nat Med, 1998. 4(9):1025-31.

14. Yonekura H, Yamamoto Y, Sakurai S, Watanabe T, Yamamoto H. Roles of the receptor for advanced glycation endproducts in diabetes-induced vascular injury. J Pharmacol Sci, 2005. 97(3):305-11.

15. Lin L. RAGE on the toll road? Cell Mol Immunol, 2006. 3(5):351-8.

16. Bierhaus A, Humpert PM, Morcos M, Wendt T, Chavakis T, Arnold B, Stern DM, Nawroth PP. Understanding RAGE, the receptor for advanced glycation end products. J Mol Med (Berl), 2005. 83(11):876-86.

17. Wautier JL, Zoukourian C, Chappey O, Wautier MP, Guillausseau PJ, Cao R. Receptor-mediated endothelial cell dysfunction in diabetic vesculopathy. Soluble receptor for advanced glycation end products blocks hyperpermeability in diabetic rats. J Clin Invest, 1996. 97(1):238-43.

18. Tanaka N, Yonekura H, Yamagishi S, Fujimori H, Yamamoto Y, Yamamoto H. The receptor for advanced glycation end products is induced by the glycation products themselves and tumor necrosis factor-alpha through nuclear factor-kappa B, and by 17 beta-estradiol though Sp-1 in human vascular endothelial cells. J Biol Chem, 2000. 275:25781-90

19. Schmidt AM, Yan SD, Wautier JL, Stern D. Activation of receptor for advanced glycation end products: a mechanism for chronic vascular dysfunction in diabetic vasculopathy and atherosclerosis. Circ Res, 1999. 84(5):489-97.

20. Chavakis T, Bierhaus A, Nawroth, PP. RAGE (receptor for advanced glycation end products): a central player in the inflammatory response. Microbes Infect, 2004. 6(13):1219-25.

21. Iwashima Y, Eto M, Horiuchi S, Sano H. Advanced glycation end product-induced peroxisome proliferator-activated receptor y gene expression in the cultured mesangial cells. Biochem Biophys Res Commun, 1999. 264(2):441-8. 
22. Järvisalo MJ, Raitakari M, Toikka JO, Putto-Laurila A, Rontu R, Laine S. Endothelial dysfunction and increased arterial intima-media thickness in children with type 1 diabetes. Circulation, 2004. 109(14):1750-5.

23. Vanhoutte PM. Related Articles, Links. Endothelial control of vasomotor function: from health to coronary disease. Circ J, 2003. 67(7):572-5.

24. Jiang C, Ting AT, Seed B. PPAR-gamma agonists inhibit production of monocyte inflammatory cytokines. Nature, 1998. 391(6662):82-6.

25. Robinson E, Grieve DJ. Significance of peroxisome proliferator-activated receptors in the cardiovascular system in health and disease. Pharmacol Ther, 2009. 122(3):246-63.

26. Hattori Y, Hattori S, Sato N, Kasai K. High-glucoseinduced nuclear factor kappaB activation in vascular smooth muscle cell. Cardiovasc Res, 2000. 46(1):188-97.

27. MOHAMED MR, MCFADDEN G. NFkappaB inhibitors: strategies from poxviruses. Cell Cycle, 2009. 8(19):3125-3132.

28. Montera VSP. Benefícios dos nutrientes antioxidantes e seus co-fatores no controle do estresse oxidativo e inflamação na insuficiência cardíaca. Rev SOCERJ, 2007. 20(1):20-27.

29. Yang J, Park Y, Zhang H, Xu X, Laine GA, Dellsperger KC, Zhang C. Feed-forward signaling of TNF-alpha and NF-kappaB via IKK-beta pathway contributes to insulin resistance and coronary arteriolar dysfunction in type 2diabetic mice. Am J Physiol Heart Circ Physiol, 2009. 296(6):H1850-8.

30. Li J, Schmidt AM. Characterization and functional analysis of the promoter of RAGE, the receptor for advanced glycation end products. J Biol Chem, 1997. 272(26):16498-506.

31. Ho E, Bray TM. Antioxidants, NF- kappaB activation, and diabetogenesis. Proc Soc Exp Biol Med, 1999. 222(3):205-13.

32. Bierhaus A, Schiekofer S, Schwaninger M, Andrassy M, Humpert PM, Chen J. Diabetes-associated sustained activation of the transcription factor nuclear-kappa B. Diabetes, 2001. 50(12):2792-808.

33. Hofmann $\mathrm{M}$, et al. Insufficient glycemic control increases nuclear factor-kappa B binding activity in peripheral blood mononuclear cells isolated from patients with type 1 diabetes. Diabetes Care, 1998. 21(8);1310-6.

34. Arnalich F, Hernanz A, López-Maderuel OD, De La Fuente M, Arnalich FM, Andres-Mateos E, FernándezCapitán C, Montiel C. Intracellular glutathione deficiency is associated with enhanced nuclear factor-kappaB activation in older non-insulin dependent diabetic patients. Free Radic Res, 2001. 35(6):873-84.

35. Chester AH, Yacoub MH. Role of oxidant stress in cytokine-induced activation of NF-Kb in human aortic smooth muscle cells. Am J Physiol, 1999. 46(5 Pt 2):H1975-84.
36. Mukhopadhyay A, Manna SK, Aggarwal BB. Pervanadate induced nuclear factor-KB activation requires tyrosinephosphorylation and degradation of $\mathrm{IkB} \alpha$. Comparasion with tumor necrosis factor- $\alpha$. J Biol Chem, 2000. 275(12):8549-55.

37. Dandona P, Aljada A, Mohanty P, Ghanim H, Hamouda W, Assian E, Ahmad S. Insulin inhibits intranuclear nuclear factor kappaB and stimulates IkappaB in mononuclear cells in obese subjects: evidence for an anti-inflammatory effect? J Clin Endocrinol Metab, 2001. 86(7):3257-65.

38. Silva CO, Colette C, Monnier L, Descomps B, Pares-Herbute N. Insulin alters nuclear factor-kB and peroxisome proliferator-activated receptor-gamma protein expression induced by glycated bovine serum albumin in vascular smooth-muscle cells. J Lab Clin Med, 2005. 145(3):144-50.

39. Golovchenko I, Goalstone ML, Watson P, Brownlee M, Draznin B. Hyperinsulinemia enhances transcriptional activity of nuclear factor-kB induced by angiotensin II, hyperglycemia, and advanced glycosylation end products in vascular smooth muscle cells. Cir Res, 2000. 87(9):74652.

40. Kliewer SA, Umesono K, Noonan DJ, Heyman RA, Evans RM. Convergence of 9-cis retinoic acid and peroxisome proliferator signalling pathways through heterodimer formation of their receptors. Nature, 1992. 358(6389):771-4.

41. Chan LS, Wells RA. Cross-talk between PPARs and the partners of RXR: a molecular perspective. PPAR Res, 2009. 2009:925309.

42. Forman BM, Tontonoz P, Chen J, Brun RP, Spiegelman BM, Evans RM. 15-deoxy-delta12, 14-prostaglandin J2 is a ligand for the adipocyte determination factor PPAR gamma. Cell, 1995. 83(5):803-12.

43. Ricote M, Glass CK. PPARs and molecular mechanisms of transrepression. Biochim Biophys Acta, 2007. 1771(8):926-35.

44. Ricote M, Huang J, Fajas L, Li A, Welch J, Najib J. Expression of the peroxisome proliferator-activated receptor gamma (PPAR- $\gamma$ ) in human atherosclerosis and regulation in macrophages by colony stimulating factors and oxidized low-density lipoprotein. Proc Natl Acad Sci USA, 1998. 95(13):7614-9.

45. Verges B. Do the pleiotropic effects of statins have a clinical significance? Arch Mal Coeur Vaiss, 2004. 97(12):1231-5.

46. Rossi A, Kapahi P, Natoli G, Takahashi T, Chen Y, Karim M, Santoro MG. Antiinflammatory cyclopentenone prostaglandins are direct inhibitors of Ik-B kinase. Nature, 2000. 403(6765):103-8.

47. Rival Y, Benéteau N, Taillandier T, Pezet M, Dupont-Passelaigue E, Patoiseau JF, Junquéro D, Colpaert F, Delhon A. PPARa and PPARg activators inhibit cytokine- induced nuclear translocation of NF-kB and expression of VCAM-1 in EAhy926 endothelial cells. Eur J Pharmacol, 2002. 435(2-3):143-51. 
48. Chai JT, Choudhury RP. Cardiometabolic interventions - focus on transcriptional regulators. Eur J Cardiovasc Med, 2013. 2(3):212-8.

49. Schiffrin EL. More evidence of cardiorenal protective effects of peroxisome proliferator-activated receptor activation. Hypertension, 2005. 46(2):267-8.

50. Tontonoz P, Nagy L, Alvarez JC, Thomazy VA, Evans RM. PPARgamma promotes monocyte/macrophage differentiation and upgrade of oxidized LDL. Cell, 1998. 93(2):241-52.

51. Lipsky RH, Eckert DM, Tang Y, Ockenhouse CF. The carboxyl-terminal cytoplasmic domain of CD36 is required for oxidized low-density lipoprotein modulation of NFkappaB activity by tumor necrosis factor-alpha. Recept Signal Transduct, 1997. 7(1):1-11.

52. Han CY, Pak YK. Oxidation-dependent effects of oxidized LDL: proliferation or cell death. Exp Mol Med, 1999. 31(4):165-73.

53. Janabi M, Yamashita S, Hirano K, Sakai N, Hiraoka H, Matsumoto K, Zhang Z, Nozaki S, Matsuzawa Y. Oxidized LDL-induced NF-kappa B activation and subsequent expression of proinflammatory genes are defective in monocyte-derived macrophages from CD36-deficient patients. Arterioscler Thromb Vasc Biol, 2000. 20(8):1953-60.

54. Chinetti G, et al. PPAR-alpha and PPAR-gamma activators induce cholesterol removal from human macrophage foam cells through stimulation of the ABCA1 pathway. Nat Med, 2001. 7(1):53-8.
55. Chawla A, Barak Y, Nagy L, Liao D, Tontonoz P, Evans RM. PPARgamma-dependent and-independent effects on macrophage-gene expression in lipid metabolism and inflammation. Nat. Med, 2001. 7(1):48-52.

56. Rosenson RS. Effects of peroxisome proliferator-activated receptors on lipoprotein metabolism and glucose control in type 2 diabetes mellitus. Am J Cardiol, 2007. 99(4A):96B-104B.

57. Aitman TJ, et al. Identification of CD36 (fat) as an insulinresistence gene causing defective fatty acid and glucose metabolism in hypertensive rats. Nat Genet, 1999. 21(1):76-3.

58. Ibrahimi A, Bonen A, Blinn WD, Hajri T, Li X, Zhong K, Cameron R, Abumrad NA. Muscle-specific overexpression of FAT / CD36 enhances fatty acid oxydation by contracting muscle, reduces plasma triglycerides and fatty acids, and increases plasma glucose and insulin. J Biol Chem, 1999. 274(38);26761-6.

59. Liang C, Han S, Okamoto H, Carnemolla R, Tabas I, Accili $\mathrm{D}$. Increased CD36 protein as a response to defective insulin signaling in macrophages. J Clin Invest, 2004. 113(5):764-73.

60. Eldor R, Defronzo RA, Abdul-Ghani M. In Vivo Actions of Peroxisome Proliferator-Activated Receptors: Glycemic control, insulin sensitivity, and insulin secretion. Diabetes Care, 2013. 36(Suppl 2):S162-74.

61. Silva CO, Delbose S, Azais C, Monnier L, Cristol JP, ParesHerbute N. Modulation of CD36 protein expression by AGEs and insulin in aortic VSMCs from diabetic and nondiabetic rats. Nutr Metab Cardiovasc Dis, 2008. 18(1):23-30. 